\title{
Low Temperature Rhombohedral Single Crystal SiGe Epitaxy on c-plane Sapphire
}

\author{
Adam J. Duzik*a and Sang H. Choi ${ }^{\text {b }}$ \\ ${ }^{a}$ National Institute of Aerospace, 100 Exploration Way, Hampton, VA, 23666 \\ bNASA Langley Research Center, 8 West Taylor St., Hampton, VA, 23681
}

\begin{abstract}
Current best practice in epitaxial growth of rhombohedral SiGe onto (0001) sapphire $\left(\mathrm{Al}_{2} \mathrm{O}_{3}\right)$ substrate surfaces requires extreme conditions to grow a single crystal SiGe film. Previous models described the sapphire surface reconstruction as the overriding factor in rhombohedral epitaxy, requiring a high temperature Al-terminated surface for high quality films. Temperatures in the $850-1100^{\circ} \mathrm{C}$ range were thought to be necessary to get $\mathrm{SiGe}$ to form coherent atomic matching between the (111) SiGe plane and the (0001) sapphire surface. Such fabrication conditions are difficult and uneconomical, hindering widespread application.

This work proposes an alternative model that considers the bulk sapphire structure and determines how the SiGe film nucleates and grows. Accounting for thermal expansion effects, calculations using this new model show that both pure Ge and $\mathrm{SiGe}$ can form single crystal films in the $450-550^{\circ} \mathrm{C}$ temperature range. Experimental results confirm these predictions, where x-ray diffraction and atomic force microscopy show the films fabricated at low temperature rival the high temperature films in crystallographic and surface quality. Finally, an explanation is provided for why films of comparable high quality can be produced in either temperature range.
\end{abstract}

Keywords: Magnetron Sputtering Deposition, Semiconductor Devices, Group IV Semiconductor Materials, Bandgap Engineering, X-ray Diffraction

\section{INTRODUCTION}

Conventional semiconductor heteroepitaxy consists of thin film deposition of two different semiconductors with the same or similar crystal structures. Common examples are InAs on GaAs (both zinc-blende crystals) and GaN on sapphire (wurtzite film on trigonal substrate). Other work has focused on producing SiGe films through conventional heteroepitaxy. However, only polycrystalline and amorphous SiGe were achieved. ${ }^{1-3}$ Single crystal SiGe films of any composition remained elusive, until recently. Work by Park et al. proved SiGe could form high quality single crystal films on c-plane sapphire, combining a traditionally diamond cubic structure onto a trigonal substrate. ${ }^{4}$ The SiGe film was found to be a trigonal crystal structure, rather than its stand-alone diamond cubic structure. This was maintained even over large thicknesses: $\sim 1 \mu \mathrm{m}$ or greater. $\mathrm{SiGe}$ grown on sapphire at $820^{\circ} \mathrm{C}$ showed a tendency to form two crystallographic twins, while SiGe films grown at $850^{\circ} \mathrm{C}$ consisted of up to $99.7 \%$ of only one twin. While promising, this novel form of thin film heteroepitaxy was only achievable at temperatures of $850^{\circ} \mathrm{C}$ or higher. These conditions are impractical for commercial device application and often not repeatable in quality. Moreover, no model was proposed describing the bonding at the interface between diamond cubic (111) plane atoms and the trigonal (0001) plane atoms. Without such a model, it is unknown whether single crystal $\mathrm{SiGe} / \mathrm{Al}_{2} \mathrm{O}_{3}$ films are possible at lower film growth temperatures. In this work, a model is developed for how $\mathrm{Si}$ and Ge bond to the (0001) sapphire surface, then it is determined which temperatures were the most conducive to forming coherent, single crystal thin films. This is followed with film growth at those temperatures, with success being measured as high (>99\%) single crystallinity and a flat, terraced surface with low root-mean-square (RMS) roughness.

*adam.j.duzik@nasa.gov; phone 1 757-864-6030 


\section{FILM/SUBSTRATE INTERFACE MODEL}

Thin films are often dominated by the strain state at the interface between film and substrate, dictating whether films grow in a two-dimensional fashion (layer-by-layer growth), a three-dimensional fashion (Volmer-Weber), or a hybrid fashion where growth is initially two-dimensional, then switches to a three-dimensional mound growth after a certain critical thickness (Stranski-Krastanov growth). Mound formation is a response to interfacial strain, alleviating the difference in lattice constants between film and substrate. Flat, uniform films grow layer-by-layer, requiring a low mismatch strain. The previous SiGe results demonstrated high crystallinity and low roughness, even in micron thick films. Hence, despite the difference in crystal structure, (111) plane SiGe atoms somehow align to form a coherent film on (0001) sapphire.

Interfacial strain ultimately arises from the difference in lattice constants and bulk crystal structure, not from surface atom arrangement. Thus, O-terminated or Al-terminated sapphire surfaces are not the determining factor in producing single crystal SiGe. While surface atomic structure affects the bonding and chemistry of the SiGe to the substrate, the film still needs to match the underlying bulk structure to achieve a low strain condition. Figure 1 compares the (0001) trigonal and (111) diamond cubic crystal structure planes from the top-down and side views. Both Si and Ge form diamond cubic structures; therefore, the atoms in that structure will be referred to as $\mathrm{Si} / \mathrm{Ge}$ atoms, to indicate either species may occupy the lattice sites. In Figure 2, the (111) Si/Ge plane most closely matches the Al double layer in the (0001) sapphire surface. Thus, $\mathrm{Si}$ and $\mathrm{Ge}$ atoms will align over the $\mathrm{Al}$ atoms in its bonding to the sapphire surface. For an O-terminated surface, $\mathrm{Si}$ will bond to three surface $\mathrm{O}$ atoms similar to that seen in $\mathrm{SiO}_{2}$, followed by another $\mathrm{Si}$ atom directly over the interfacial $\mathrm{Si}$ atom to satisfy the four-fold Si coordination.



Figure 1. Top-down (upper) and side (lower) views of the trigonal sapphire surface (left) vs. diamond cubic silicon (right). Patterned atoms with lighter color are more towards the surface than darker colored filled atoms. Si atoms are closer in size and spacing to the sapphire Al atoms and will mimic those atoms most upon bonding to the sapphire surface. 
Moreover, the model must account for unequal thermal expansion of bond lengths in both the substrate and the film. Related work on $\mathrm{CdTe} / \mathrm{Al}_{2} \mathrm{O}_{3}$ showed substrate temperatures of $460^{\circ} \mathrm{C}$ were ideal for forming single crystal $\mathrm{CdTe} / \mathrm{Al}_{2} \mathrm{O}_{3}{ }^{5,6}$ Below $420^{\circ} \mathrm{C}, \mathrm{CdTe} / \mathrm{Al}_{2} \mathrm{O}_{3}$ films were of poor quality, as were films grown much above $460^{\circ} \mathrm{C}$, suggesting a growth temperature window where thermal expansion of the film and substrate reduces the mismatch strain low enough to achieve coherent, high-quality films. Finding the right temperature for growth is the first major challenge in producing single crystal $\mathrm{SiGe} / \mathrm{Al}_{2} \mathrm{O}_{3}$ films.

The second major challenge to single crystal $\mathrm{SiGe} / \mathrm{Al}_{2} \mathrm{O}_{3}$ films is twin formation. Previous $\mathrm{SiGe} / \mathrm{Al}_{2} \mathrm{O}_{3}$ work showed $\mathrm{SiGe}$ can form two different crystallographic twins as evidenced in the XRD $\varphi$ angle scans showing six $\{220\}$ peaks instead of three. ${ }^{4}$ Often, three of the peaks are more intense, indicating a predominant major twin. Three less intense peaks from the minority twin appear at exactly $60^{\circ}$ between the major twin peaks. Thus, the twins are crystallographically the same, but rotated $60^{\circ}$ with respect to each other. Dominance of one twin over the other was attributed to differences in surface site activation energies for diffusion, with the dominant twin forming on atomic sites with higher activation energies. ${ }^{4}$

With these observations in hand, the models were constructed as shown in Figure 2. In these models, the twins in Figure 2a-c were arbitrarily called "up" twins. This designation arose from the fact the upper $\mathrm{Si} / \mathrm{Ge}$ atoms [checkered green, Figure 2a-c] bond to the three lower interfacial $\mathrm{Si} / \mathrm{Ge}$ atoms to form a virtual triangle (as indicated in green in the figure) that points upward. When this bonding arrangement is rotated $60^{\circ}$ within the surface plane, the triangle points downward. For these two valid rotations, the lower level atoms may then align over one of three types of sites: over the $\mathrm{Al}$ sites closer to the surface, over the $\mathrm{Al}$ atoms further from the surface, and the "blank" sites, that is, the sites where $\mathrm{Al}$ atoms are much further into the sapphire crystal. Hence, six possible configurations are possible, three for each twin. Each is incompatible with the other, and will form twin boundaries if one or more nucleate on the sapphire surface during growth. However, of the three up twins, one site type will be energetically preferable to the other two. Any incoming atoms in the higher energy sites will diffuse out of those sites into the lowest energy ones. A similar argument holds for the down twins, reducing the total number of relevant twin configurations to two. Which sites are energetically preferred is unknown, requiring nudged elastic band density functional theory simulations to accurately calculate the activation energies. This is outside the scope of this work, but does not affect the final result as the three different sapphire sites are the same distance apart. The thermal strain on the SiGe film will be the same regardless of which sites are energetically most favorable. Whichever particular configuration is lowest in energy, it results in the same lower temperature single crystal $\mathrm{SiGe} / \mathrm{Al}_{2} \mathrm{O}_{3}$ growth.

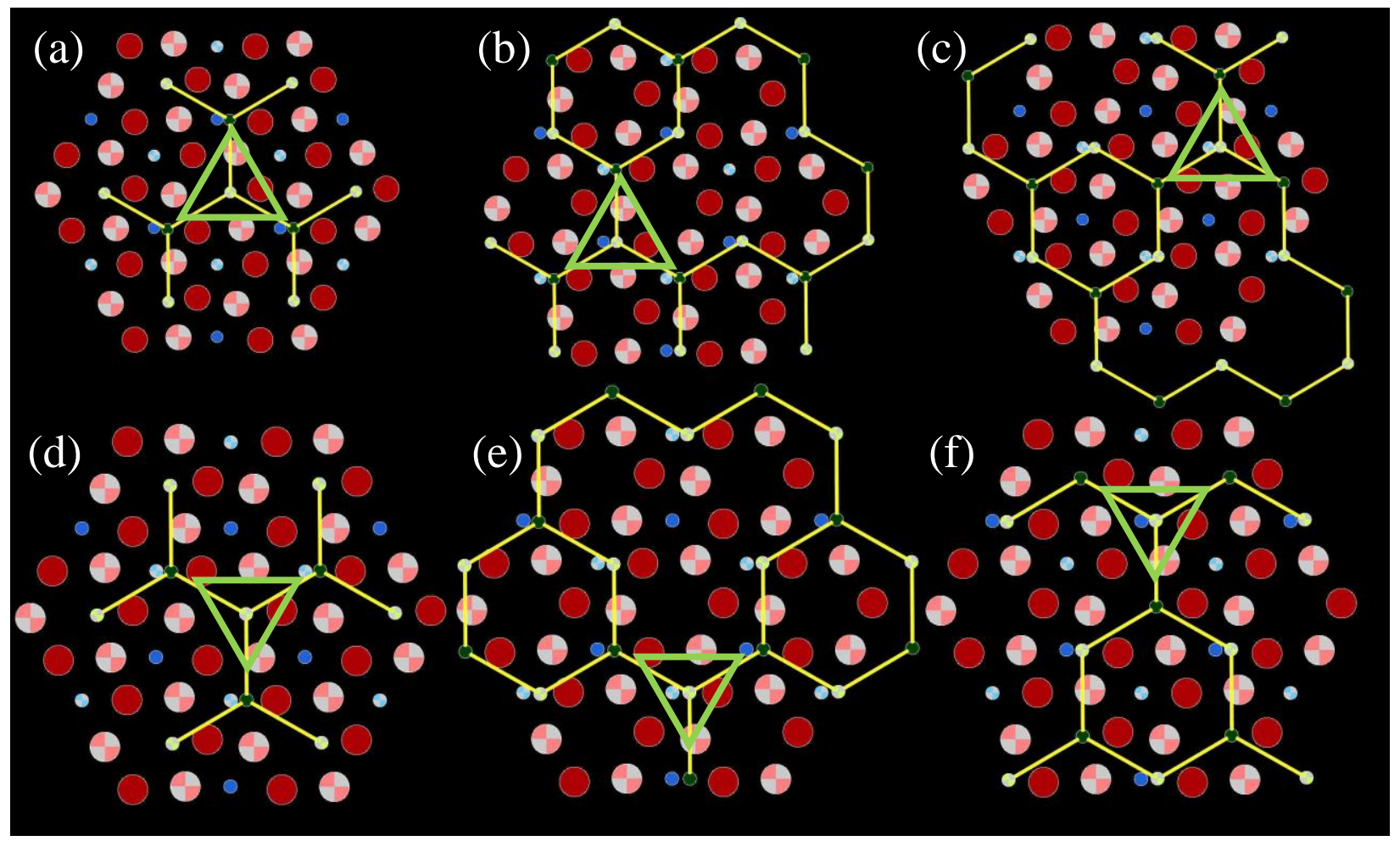

Figure 2. Overlay models of (111) planes of Si/Ge atoms (connected by yellow lines) onto the (0001) sapphire plane. The green triangle in each model marks out the "central" Si/Ge atom. The three Si/Ge atoms bonded to this atom are the interfacial 
atoms that form the virtual triangle. Si/Ge atoms are shown with bonds drawn and slightly offset to the right of the sapphire Al atoms. The three possible "up" twins (a-c) and the three possible "down" twins (d-f) can form on the (0001) sapphire plane.

\section{SIMULATION SETUP}

The calculation of the ideal temperature for forming single crystal $\mathrm{SiGe} / \mathrm{Al}_{2} \mathrm{O}_{3}$ is now considered. Comparing the (111) SiGe plane to the (0001) $\mathrm{Al}_{2} \mathrm{O}_{3}$ plane, the interfacial (111) plane atoms most closely match the spacing between the topmost $\mathrm{Al}$ atoms in (0001) $\mathrm{Al}_{2} \mathrm{O}_{3}$. The assumption here was when the SiGe spacing matches the $\mathrm{Al}$ spacing, the film was coherent, forming a stable rhombohedral crystal structure. All simulated film atom coordinates were extrapolated from the diamond cubic unit cell, using linear interpolation of bond spacing and lattice constants for mixed SiGe alloy films. From this, two parameters were defined: bond angle distortion and bond length distortion.

The first parameter represented the level of extension or compression of the cubic unit cell along its body diagonal. As either happens, the unit cell deviated from its cubic shape to form a rhombohedral unit cell, where the corner angles were no longer $90^{\circ}$. An elongation along the body diagonal, defined as a positive bond angle distortion, contracted the atoms in the (111) plane together and distended them in the direction normal to the (111) plane; a compression along the body diagonal, defined as a negative bond angle distortion, had the reverse effect. The second parameter was defined as a multiplier on the unit cell lattice constant. Values between 0 and 1 represented a contraction of the bond lengths, while any value greater than 1 represented a bond length extension.

Unit cells for SiGe were constructed for each combination of the angle and bond length distortion parameters, representing the hypothetical film crystal structure at room temperature. The thermal expansion for both the film and $\mathrm{Al}_{2} \mathrm{O}_{3}$ substrate were calculated for the temperature range of 20 to $1250^{\circ} \mathrm{C}$. At each temperature, the (111) plane SiGe interfacial atom spacings were compared to the (0001) $\mathrm{Al}$ atom spacing in $\mathrm{Al}_{2} \mathrm{O}_{3}$, and if the difference between the two was within 0.0001 angstroms, the film was declared a match to the substrate.

\section{SIMULATION RESULTS}

Running this comparison for bond angle values from -0.50 to 0.50 and bond length multipliers from 0.01 to 2 , it became apparent these parameters were not independent. Only certain combinations of these two parameters were even capable of matching the film to the substrate at any temperature, as plotted in Figure 3a-c. This was not surprising as an increase in the length multiplier can have the opposite effect of the contraction caused by an increase in bond angle distortion. Fitting a $4^{\text {th }}$ order polynomial to these parameters, the bond length multiplier needed to match the film to the substrate for any given bond angle distortion was predictable. This reduced the distortion necessary to match $\mathrm{SiGe}$ to $\mathrm{Al}_{2} \mathrm{O}_{3}$ to a single parameter.

Plots of the temperatures where thermal expansion caused a lattice match are given in Figure 3d-f for pure Si, pure Ge, and $\mathrm{Si}_{0.15} \mathrm{Ge}_{0.85}$. Local minima in these curves (circled) indicate the lowest temperatures of matching, corresponding to where the lattice mismatch and subsequent film strain are minimized and thus which SiGe crystal structure forms. Based on the minima found in Figure 3, Ge forms a strong match around $450^{\circ} \mathrm{C}$ and $\mathrm{Si}_{0.15} \mathrm{Ge}_{0.85}$ does the same around $500^{\circ} \mathrm{C}$. These are the wafer surface temperatures; in actual experiments the reported growth temperature is from a thermocouple that sits beneath the wafer plate. Thus, the experimental setpoint was higher than the matching temperature to account for differences in reported thermocouple temperature and actual wafer surface temperature. 


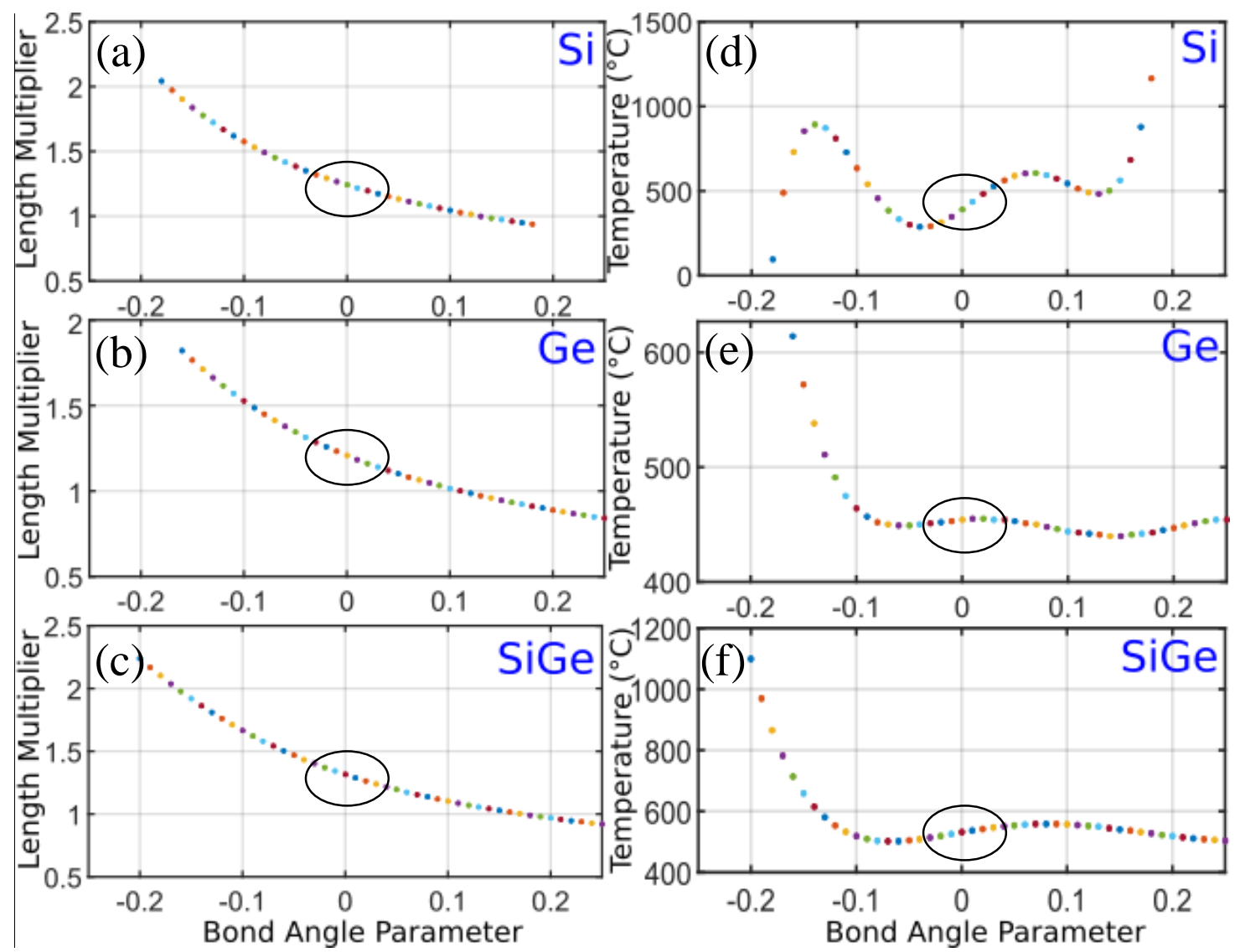

Figure 3. Plots of film distortion needed to match the underlying sapphire substrate for (a) Si, (b) Ge, and (c) Si $i_{0.15} G e_{0.85}$ films. Regions of lowest mismatch and thus highest likelihood of formation are circled. Temperature (in $\left.{ }^{\circ} \mathrm{C}\right)$ required for film/substrate matching are plotted for (d) Si, (e) Ge, and (f) Sio.15Geo.85 films.

\section{EXPERIMENTAL SETUP}

All experiments were done in a SRN-150 magnetron sputtering system. 50mm sapphire wafers, oriented to the (0001) plane as the epitaxial growth surface, were loaded on a Mo holder plate, with the epi-ready side facing upward. $175 \mathrm{~mm} \mathrm{Si}$ and Ge targets facing downward were used to sputter thin films onto the sapphire wafer substrate. Ar was used as the sputtering plasma, held at a constant pressure of 5 mtorr during growth and at a flow rate of $50 \mathrm{sccm}$. Substrate temperatures ranging from $400^{\circ} \mathrm{C}$ to $560^{\circ} \mathrm{C}$ were achieved with a quartz heater bulb assembly, consisting of six bulbs beneath the wafer holder plate. The temperature was measured by a thermocouple in the center of the assembly.

Film thicknesses and composition were estimated from a series of calibration samples. Pure Si or pure Ge films were deposited on a series of wafers at $550^{\circ} \mathrm{C}$ at $\mathrm{RF}(\mathrm{Si})$ or $\mathrm{DC}(\mathrm{Ge})$ powers ranging from $100-800 \mathrm{~W}$, and all depositions were 1 hour long. The resulting wafers were removed, cleaved, and the cross-sectional film thickness measured with a JEOL 6360 scanning electron microscope. From this, the sputtering rates were easily obtained. With the resulting thicknesses at each sputtering power, the SiGe film thicknesses were taken to be the addition of the $\mathrm{Si}$ and Ge thicknesses. With this in hand, the SiGe compositions were estimated as the ratio of individual $\mathrm{Si}$ and Ge thicknesses to the combined SiGe thickness. A SiGe film has two requirements for use in device fabrication. First, it must be a monocrystalline film, that is, one twin must comprise $99 \%$ or more of the film. Second, the surface must have a flat, terraced morphology. X-ray diffraction (XRD) and atomic force microscope (AFM) methods were used to establish these two qualities in each film.


constant goniometer position. The $2 \theta-\omega$ scans were done from $5-120^{\circ}$ to establish overall film quality through measurement of the full-width at half-maximum (FWHM) and peak intensity. The $\varphi$ axis is parallel with the (111) SiGe surface normal, and each scan completed one whole revolution of the wafer at a constant position designed to find all the $\{220\}$ reflections. A similar $\varphi$ scan is done with the sapphire (0001), parallel with the (111) SiGe surface, to find all the 
$\{10 \overline{1} 4\}$ reflections. A high quality SiGe film will have three peaks in the SiGe scan, corresponding to the three $\{220\}$ planes in the film. The presence of six peaks, $60^{\circ}$ offset from one another, corresponds to two twins, rotated $60^{\circ}$ with respect to one another, each with three $\{220\}$ reflections. Relative peak heights of these twins are compared to determine how much of each twin exists in the SiGe film. Finally, two XY area maps of a (220) reflection, one for each twin, were done to find the distribution of both twins over the film, whether they are evenly distributed or segregated. This was done after the $\varphi$ scan when the $\varphi$ angle positions were known. Setting the goniometer to one of these positions led to a constant illumination of that (220) plane, mapping the location of that twin over the whole wafer as the XY scan proceeded.

Surface film quality was established with a Quesant Q-scope AFM. Scans $10 \times 10 \mu \mathrm{m}^{2}$ in size were taken at the center of each wafer and the root-mean-square (RMS) roughness calculated over the whole image. Rough films (not shown) tended to have rounded mounds on the surface, while better films showed flatter, terraced surfaces.

\section{EXPERIMENTAL RESULTS}

The results of $\mathrm{SiGe} / \mathrm{Al}_{2} \mathrm{O}_{3}$ film growth at $450^{\circ} \mathrm{C}$ are shown in Figure 4 and Figure 5. The AFM image of Figure 4 reveals a number of small, irregular-shaped terraces, but overall is free of large mounds of droplets. The XRD $\varphi$ scan data of Figure 5a shows a significant (1\%) fraction of minor twins formed during growth. Figure $5 \mathrm{~b}$ shows a $2 \theta-\omega$ scan with a high intensity $\left(162094.3\right.$ counts) and narrow (FWHM $0.017^{\circ}$ ) peak at $27.53^{\circ}$, the location of the Ge peak. This indicates a high-quality, uniform film along the wafer axial direction. The double peak at $90^{\circ}$ belongs partly to $\mathrm{SiGe}$ and partly to sapphire. The left peak at $89.85^{\circ}$ is the $\mathrm{SiGe}(511)$ peak, while the right peak at $90.95^{\circ}$ is the $\mathrm{Al}_{2} \mathrm{O}_{3}(00012)$ peak. Figure $5 \mathrm{c}$ and $\mathrm{d}$ are the wafer maps of the major and minor twin distributions on the wafer, respectively. The major twin coverage is somewhat uniform, with the highest concentration in the lower left portion of the wafer. Conversely, the minor twin is mostly concentrated on the upper left edge of the wafer, suggesting an invisible contamination existed before growth. Despite this, the Ge film is still coherent with the underlying sapphire atomic structure, indicating the $\mathrm{SiGe} / \mathrm{Al}_{2} \mathrm{O}_{3}$ deposition is a robust process, dictated solely by the alignment of $\mathrm{Si}$ and $\mathrm{Ge}$ atoms to the underlying $\mathrm{Al}$ and $\mathrm{O}$ sublattices. While surface cleanliness is vital, it is not the root cause for rhombohedral SiGe formation; otherwise, this process would be much more inconsistent.

The encouraging results of $\mathrm{SiGe}$ deposition at $450^{\circ} \mathrm{C}$ lend great credence to the above model of diamond cubic (111) matching to the (0001) rhombohedral surface. As such, $\mathrm{Si}_{0.15} \mathrm{Ge}_{0.85}$ is predicted to be best matched around $500^{\circ} \mathrm{C}$. Growing at this temperature produced the surface shown in Figure 6. Like Ge, the terraces are flat and smooth, but unlike the $\mathrm{SiGe} / \mathrm{Al}_{2} \mathrm{O}_{3}$ case, the terraces retain a much more regular triangular shape. This surface is also usable for device production. The corresponding XRD results of Figure 7 show a very high quality film. Figure 7a is a plot of the $\varphi$ scan data of the $\mathrm{Si}_{0.15} \mathrm{Ge}_{0.85}\{220\}$ positions overlaid with the sapphire $\{10 \overline{1} 4\}$ positions, revealing almost no minor twin formation during growth. Figure $7 \mathrm{~b}$ shows the $2 \theta-\omega$ scan of the SiGe surface, with an intensity at the SiGe (111) peak of 724464.8 counts and a FWHM of $0.165^{\circ}$, showing $\mathrm{SiGe} / \mathrm{Al}_{2} \mathrm{O}_{3}$ can be produced at production grade uniformity for device fabrication. Figure $7 \mathrm{c}$ and $\mathrm{d}$ show the major twin coverage is uniform over the surface, with the only real source of minor twins arising from the wafer touch points at the bottom of the wafer in Figure 7d. Improved wafer handling would eliminate this problem. 


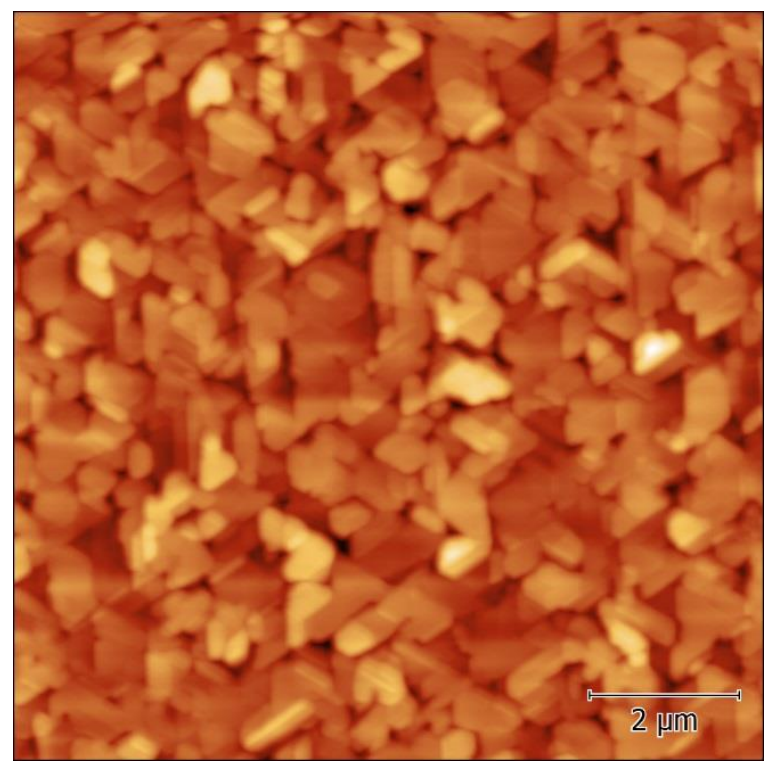

Figure 4. $10 x 10 \mu \mathrm{m}^{2}$ AFM image of the Sio.15Ge0.85 film surface after growth at $450^{\circ} \mathrm{C}$ thermocouple temperature. The surface consists of flat, triangular-shaped terraces rather than the mounds seen in other samples. RMS roughness is $7.04 \mathrm{~nm}$. 

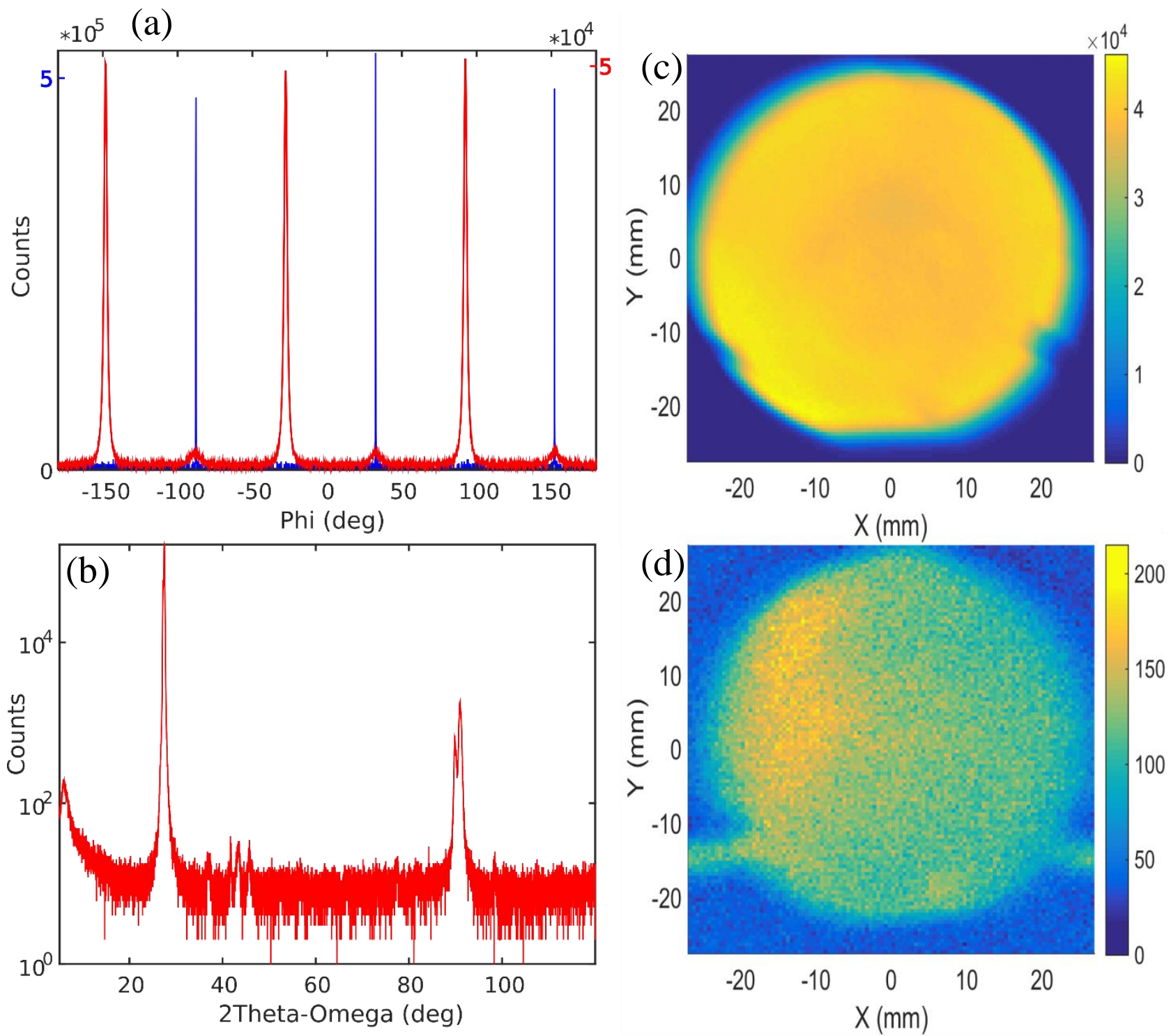

Figure 5. XRD of $\mathrm{SiGe}_{\mathrm{A}} \mathrm{Al}_{2} \mathrm{O}_{3}$ grown at $450^{\circ} \mathrm{C}$. (a) $\varphi$ scans of $\{220\} \mathrm{Si}_{0.15} \mathrm{Ge} e_{0.85}$ (red) and $\{10 \overline{1} 4\}$ sapphire (blue) planes showing $0.2 \%$ minor twin formation occurred during growth. (b) $2 \theta$ - $\omega$-omega scan showing the (111) SiGe peak at $27^{\circ}$ with a high intensity and FWHM of $0.17^{\circ}$. Wafer map scans of (c) the major twin and (d) the minor twin, showing the majority of the defects clustered to one side during growth. 


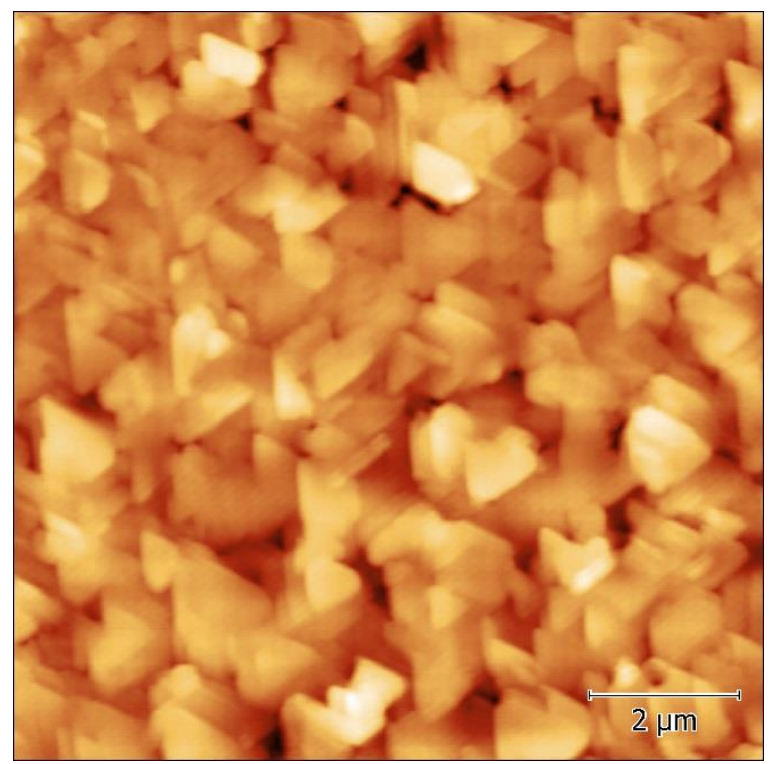

Figure 6. $10 \times 10 \mu \mathrm{m}^{2}$ AFM image of a Sio.15Ge0.85 film surface after growth at $500^{\circ} \mathrm{C}$. Surface is very flat, the triangular terraces produce only a $R M S$ roughness of $4.04 \mathrm{~nm}$. 

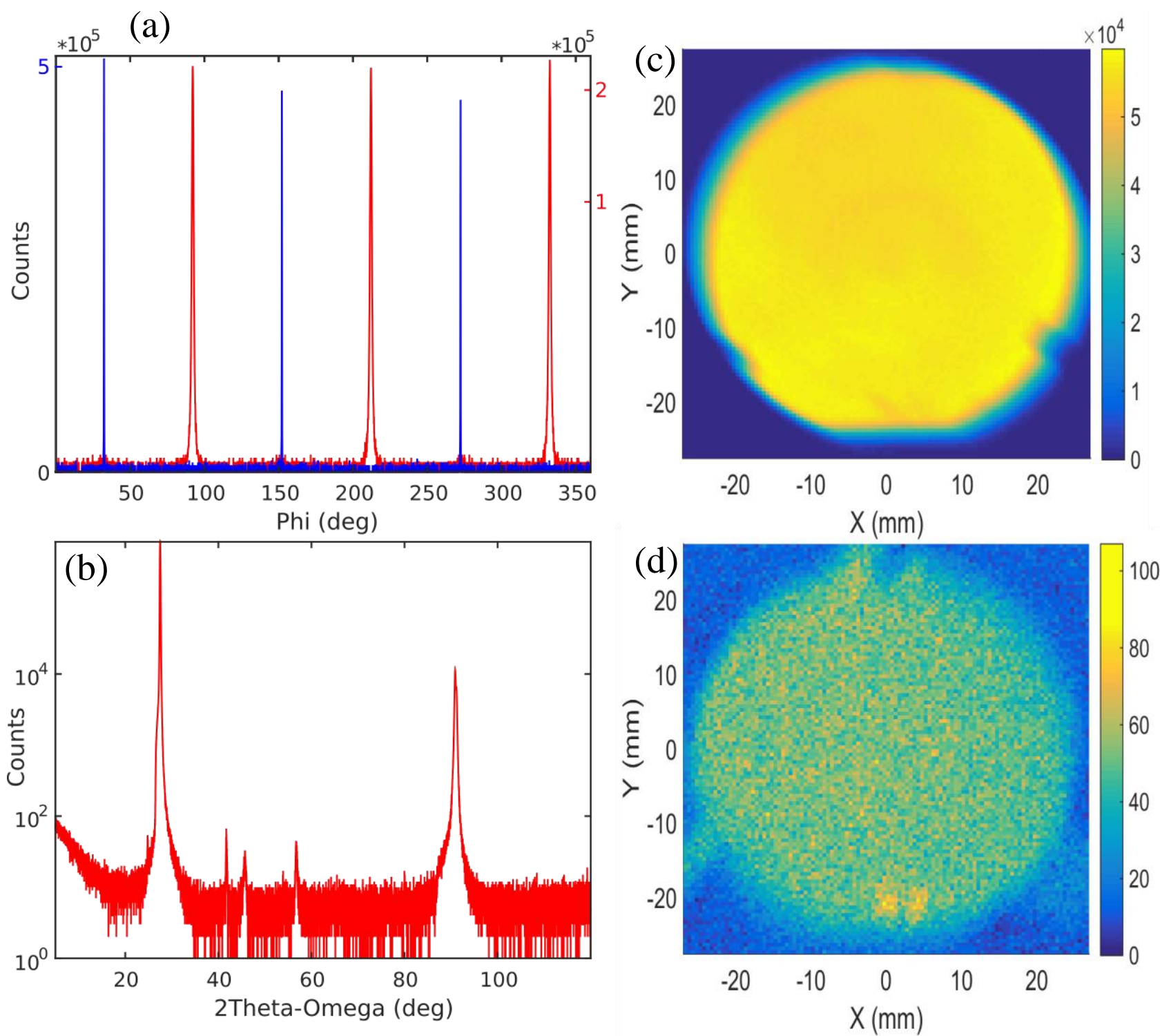

Figure 7. XRD of $\mathrm{SiGe}_{\mathrm{A}} \mathrm{Al}_{2} \mathrm{O}_{3}$ film grown at $500^{\circ} \mathrm{C}$. (a) $\varphi$ scan of $\{220\} \mathrm{Si}_{0.15} \mathrm{Ge}_{0.85}$ (red) and $\{10 \overline{1} 4\}$ sapphire (blue) planes, with a SiGe single crystallinity of $99.9 \%$. The sapphire planes align with the minor twin $\{220\}$ positions. (b) $2 \theta-\omega$ scan of the SiGe film, showing high intensity, narrow peaks at 27 and $90^{\circ}$. Distribution of $(c)$ major twin and (d) minor twin. Major twin coverage is uniform, while the minor twins are concentrated at touch points of the wafer tweezers used during handling.

\section{DISCUSSION}

Despite these promising results for $\mathrm{SiGe} / \mathrm{Al}_{2} \mathrm{O}_{3}$ at $450^{\circ} \mathrm{C}$ and $\mathrm{SiGe} / \mathrm{Al}_{2} \mathrm{O}_{3}$ at $500^{\circ} \mathrm{C}$, some issues still remain. Efforts to reproduce these films have been only partially successful. Successive wafer growths at the same conditions have not produced the flat terrace structure, but instead shows a mix of terraces and droplets. XRD scans of each film show a consistent achievement of $\sim 98 \%$ monocrystallinity. While encouraging, it is not sufficient for device growth. The wafer maps in Figure 5d and Figure 7d show several small spots where a minor twin signal was recorded. This suggests the interface that forms during the initial stages of deposition is of paramount importance. One possible explanation of minor twin formation lies in surface contamination. The touch point defects in Figure $7 \mathrm{~d}$ imply even the smallest of particles have a much bigger impact than in traditional heteroepitaxy. High quality III-V heteroepitaxy is common even after loading 
a substrate sitting in air, as the film has the same crystal structure as the substrate; only bond length differences need to be overcome. For rhombohedral epitaxy, the cleanliness requirement is much stricter to bridge the difference in crystal structure and bond length. Another possibility exists in surface kinetics. Park et al. proposed one twin dominates over the other due to differences in diffusion barrier energies; the major twin rests on sites with deep energy wells, while minor twins start on atomic sites with much smaller energy wells. ${ }^{4}$ Adatoms will diffuse out of minor twin sites into major twin sites, provided sufficient time is allowed for this to occur. If the deposition rate is high enough or the substrate surface temperature is low enough, the minor twins can be "frozen" in place before diffusion eliminates them. An interesting future study would focus on the growth of a single SiGe monolayer with molecular beam epitaxy, after which the film is annealed, followed by complete film growth. This would discriminate between surface contamination and kinetics as the cause of minor twin formation.

\section{CONCLUSION}

An atomistic model for $\mathrm{SiGe} / \mathrm{Al}_{2} \mathrm{O}_{3}$ growth was proposed and verified experimentally. $\mathrm{Si}$ and $\mathrm{Ge}$ atoms were shown to prefer alignment over the $\mathrm{Al}$ subsurface sites in the $\mathrm{Al}_{2} \mathrm{O}_{3}$ structure, indicating bulk $\mathrm{Al}_{2} \mathrm{O}_{3}$ structure, not $\mathrm{Al}$ or $\mathrm{O}$ surface termination, is the deciding factor in rhombohedral SiGe formation. Based on these predictions, a $\mathrm{SiGe} / \mathrm{Al}_{2} \mathrm{O}_{3}$ film was deposited at $450^{\circ} \mathrm{C}$ and at $500^{\circ} \mathrm{C}$ in light of the model. Both films showed high monocrystallinity, with $99.8 \%$ and $99.9 \%$, respectively, of the films consisting of one twin. AFM showed a low roughness for both films, with flat terraces dominating both surfaces. XRD wafer maps show the much stricter necessity for surface cleanliness over traditional heteroepitaxy. Such films are promising for semiconductor device fabrication.

\section{REFERENCES}

[1] Osten, H. J., Klatt, J., Lippert, G., Bugiel, E., Hinrich, S., "Two-dimensional lattice-mismatched heteroepitaxy of germanium on silicon beyond the critical thickness by introducing a surfactant," Appl. Phys. Lett. 60(20), 25222524 (1992).

[2] Cullis, a. G., Booker, G. R., "The epitaxial growth of silicon and germanium films on (111) silicon surfaces using UHV sublimation and evaporation techniques," J. Cryst. Growth 9, 132-138 (1971).

[3] Saraswat, K. C., King, T. J., "Low temperature germanium-silicon on insulator thin-film transistor," US5250818 A, USPTO (1993).

[4] Park, Y., King, G. C., Choi, S. H., "Rhombohedral epitaxy of cubic SiGe on trigonal c-plane sapphire," J. Cryst. Growth 310(11), 2724-2731 (2008).

[5] Glass, H. L., Appleby Woods, M. R., Buehnerkemper, M. L., Varnum, D. L., Weismuller, T. P., "Control of microtwins in MOCVD of CdTe on sapphire," J. Cryst. Growth 128(1-4), 617-621 (1993).

[6] Kasuga, M., Futami, H., Iba, Y., "Vapor phase epitaxy of CdTe on sapphire and GaAs," J. Cryst. Growth 115(14), 711-717 (1991). 